\title{
O conceito de risco e os seus efeitos simbólicos nos acidentes com instrumentos perfurocortantes
}

\author{
The concept of risk and its symbolic effects in accidents with sharp instruments \\ El concepto de riesgo y sus efectos simbólicos en accidentes con instrumentos perfurocortantes
}

\begin{abstract}
Everaldo José da Silva', Maria da Glória Lima", Maria Helena Palucci Marziale"II
' Universidade de Brasília, Coordenação de Atenção à Saúde. Brasília-DF, Brasil.

"Universidade de Brasília, Departamento de Enfermagem, Área de Administração em Serviços de Enfermagem. Brasília-DF, Brasil.

II' Universidade de São Paulo, Escola de Enfermagem de Ribeirão Preto, Departamento de Enfermagem Geral e Especializada.
\end{abstract} Ribeirão Preto-SP, Brasil.

Submissão: 30-05-2011 Aprovação: 26-11-2012

RESUMO

O estudo objetivou compreender o risco ocupacional como um processo simbólico que estrutura as atividades diárias dos profissionais da área de saúde que manipulam instrumentos perfurocortantes. Pesquisa descritiva realizada em um hospital universitário com dados coletados por meio do levantamento dos acidentes de trabalho no Centro de Tratamento Intensivo adulto e na Lavanderia, pela aplicação de um questionário a 105 profissionais de saúde. Os dados foram analisados por meio de uma perspectiva interdisciplinar para conciliar a análise quantitativa e qualitativa dos dados. Os resultados revelaram que, embora os dados indiquem baixo índice de acidentes, ainda é preciso aprimorar instrumentos como o mapa de riscos e o uso de equipamentos de segurança, bem como investir na capacitação das equipes.

Descritores: Riscos Ocupacionais; Equipamentos de Segurança; Acidentes de Trabalho.

ABSTRACT

The study aims to understand the occupational risks as symbolic processes that shapes the daily activities of health professionals that manipulate sharps objects. Descriptive research was conducted at a university hospital, with information collected through the survey on the accidents at work in Intensive Care and hospital laundry, with the application of 105 questionnaires. The information was analyzed through an interdisciplinary perspective, and enabled the conciliation of quantitative and qualitative analysis. The results revealed that although the number of accidents is low, it is still necessary to improve the instruments such as the risk map, the use of safety equipment and staff training.

Key words: occupational risks, safety equipment, work accidents.

\section{RESUMEN}

El estudio tuvo como objetivo comprender los riesgos profesionales en los procesos simbólicos que dan forma a las actividades diarias de los trabajadores de salud que manipulan instrumentos cortantes. Investigación descriptiva realizada en un hospital universitario de con los datos recogidos a través de la encuesta del accidentes de trabajo en la Unidad de Cuidados Intensivos y en el Servicio de lavandería, mediante la aplicación de 105 cuestionarios. Los datos fueron analizados con una perspectiva interdisciplinaria, para una posible conciliación de las análisis cuantitativa y cualitativa. Los resultados revelaron que, aunque los datos indican bajas tasas de accidentes, se necesita mejorar instrumentos tales como el mapa de riesgo, el uso de equipo de seguridad y capacitación del personal.

Palabras clave: riesgos laborales; equipo de seguridad; accidentes de trabajo. 


\section{INTRODUÇÃO}

Os trabalhadores de saúde exercem suas atividades laborais em ambientes envoltos a riscos ocupacionais, os quais podem causar-lhes adoecimento e/ou acidentes de trabalho.

Acidente do trabalho é aquele que ocorre pelo exercício do trabalho, a serviço da empresa ou pelo exercício do trabalho dos segurados especiais, provocando, direta ou indiretamente, lesão corporal, doença ou perturbação funcional que cause a morte ou a perda ou redução, permanente ou temporária, da capacidade para o trabalho ${ }^{(1)}$.

Risco é definido por toda e qualquer possibilidade de que algum elemento ou circunstância existente num dado processo ou ambiente de trabalho possa causar dano à saúde, seja por meio de acidentes, doenças ou do sofrimento dos trabaIhadores, ou ainda por poluição ambiental(2).

Um dos pontos necessários para diminuir o risco e redimensionar o papel de cada um na situação de risco, é a criação do mapa de riscos. Como é estabelecido pela NR 5, uma das obrigações da Comissão Interna de Prevenção de Acidentes (CIPA) é "elaborar o mapa de risco com assessoria do Serviço Especializado em Engenharia de Segurança e em Medicina do Trabalho (SESMT), onde houver"(3).

No caso do mapa de risco, é de fundamental importância que o trabalhador participe de todo o processo de elaboração e de execução deste instrumento de prevenção. É totalmente improdutivo e ineficaz colocar o mapa de risco nos locais necessários estabelecidos por lei se os trabalhadores não sabem lê-lo ou se não reconhecem seu valor enquanto um mecanismo de controle eficaz dos riscos.

No caso do campo de trabalho deste estudo, os hospitais, e do grupo focalizado, os profissionais que trabalham na área de saúde, é importante deixar claro a importância de prestar a atenção cotidianamente ao mapa de riscos.

Nos hospitais, o mapa de riscos deve ser afixado em locais visíveis e que orientem os profissionais a agirem em um lugar que necessite de maior atenção. A obrigatoriedade legal da elaboração do mapa de riscos é considerada um dos mais importantes elementos técnicos para a segurança no trabalho.

O mapeamento de riscos no Brasil surgiu por meio da Portaria $n^{\circ} 5$ de 17/08/92, do Departamento Nacional de Segurança e Saúde do Trabalhador ${ }^{(4)}$, sendo modificada pela Portaria ${ }^{\circ} 25$ de 29/12/94, tornando obrigatória sua elaboração pela Comissão Interna de Prevenção de Acidentes. Sua regulamentação está presente na $\mathrm{NR} \mathrm{n}^{\circ} 5$, no seu anexo $\mathrm{IV}^{(3)}$.

Os riscos ocupacionais são tratados ainda nas Normas Regulamentadoras. Na NR 9, eles são incluídos no Programa de Prevenção de Riscos Ambientais (PPRA) e a NR 32 trata dos riscos ocupacionais dos trabalhadores de instituições de saúde.

No entanto, além da vigência de leis e normas, há necessidade de compreender a atuação dos profissionais da saúde diante de uma situação de risco ocupacional para que ações preventivas possam ser efetivadas.

Um dos principais problemas apontados pelos estudiosos das áreas de saúde e de ciências humanas sobre a situação de risco no ambiente de trabalho, é o medo e a culpabilização pelo acidente ${ }^{(5)}$. A culpabilização e a responsabilização dos trabalhadores pelos acidentes no ambiente de trabalho acabam fragilizando psicologicamente os profissionais que vivem em uma situação de risco nos seus ambientes de trabalho, o que ocasiona, por sua vez, a não notificação dos acidentes.

Estudo realizado com trabalhadores de enfermagem identificou que a falta de informação quanto aos riscos, aspectos epidemiológicos e jurídicos, somado às condições laborais impostas, a falta de tempo e o receio em perder o emprego contribuem para a subnotificação de acidentes de trabalho ${ }^{(6)}$.

Outro aspecto relevante é a falta de informação dos trabalhadores sobre a identificação do risco, os procedimentos de segurança e a utilização dos Equipamentos de Proteção Coletivos (EPCs) e Individuais (EPIs).

A percepção do que é uma situação de risco está diretamente vinculada com vários fatores e não deve ser unicamente pensada com algo que está restrito ao ambiente de trabalho, pois também está presente no cotidiano dos profissionais de várias áreas por meio da configuração do trabalho na sociedade neoliberal: salário, horas de trabalho, alimentação, lazer, etc. Conforme descrição de autor ${ }^{(7)}$ que, no seu estudo, ainda acrescentou moradia e vestimenta, o indivíduo que vende sua força de trabalho para poder ganhar um salário e suprir suas necessidades, se vê explorado, desvalorizado e imbecilizado pelas relações trabalhistas atuais.

A antropóloga Mary Douglas ${ }^{(8)}$ em seu livro, La Aceptabilidad Del Riesgo según lás Ciencias Sociales, mostra como a percepção do risco faz parte de um âmbito cultural que define de um ponto de vista estratégico e, por isso, político: - o que deve ser temido e como as pessoas devem reagir diante do perigo. Um dos principais conceitos utilizados pela autora é o de "imunidad subjetiva".

As pessoas tem um forte, mas injustificado, sentido de imunidade subjetiva e quando realizam atividades muito familiares há a tendência em minimizar a probabilidade de maus resultados. Aparentemente, são subestimados os riscos que este considera controlados. A pessoa acredita que pode lidar com situações familiares e subestima os riscos inerentes aos acontecimentos que ocorrem raramente, ou, como afirmam Jaspars y Hewstone as pessoas costumam acreditar que o grave não é frequente e o comum não é grave ${ }^{(9)}$.

As discussões sobre "imunidad subjetiva" possibilitam questionar a desumanização das análises propostas sobre a "teoria do risco". Tal pressuposto está diretamente vinculado a hipótese de que a reação das pessoas e a sua percepção do risco estão diretamente vinculadas com âmbitos subjetivos e culturais.

Ao negligenciar o "mapa de riscos" e os "equipamentos de proteção individual e/ou coletivo" as pessoas estão, na verdade, reafirmando sua confiança em si mesmas. Quando alguém afirma: "Isso não acontecerá comigo!" ou "Foi apenas um descuido, isso não voltará a acontecer!". Elas estão criando estratégias discursivas para lidar com o risco e para minimizar o perigo.

Há lugares perigosos e lugares impuros que devem ser limpos e purificados por meio de rituais distintos e muitas vezes, 
esta limpeza passa pela negação do perigo ou pela negligência diante dos riscos ${ }^{(8)}$.

Para que o profissional da área de saúde encarregado de amenizar as situações de risco, (seja ele da CIPA ou não) é necessário que tenha claro que o "mapa de riscos" e outros mecanismos de controle, só serão eficazes se eles fizerem parte do contexto simbólico dos profissionais que atuam no ambiente de trabalho em questão.

A notificação do acidente de trabalho é uma prática realizada no hospital onde foi realizado este estudo, Hospital Universitário de Brasília, por meio da Comunicação Interna de Acidente de Trabalho (CIAT), e também do formulário eletrônico da Rede Eletrônica de Prevenção de Acidentes de Trabalho (REPAT/USP).

A REPAT/USP é uma rede colaborativa de pesquisas e intercâmbio de informações composta por 14 hospitais de várias regiões do país, pesquisadores e especialistas em saúde do trabalhador e que tem como finalidade o controle e a elaboração de estratégias de prevenção de acidentes de trabalho com exposição a material ${ }^{(10)}$.

No Brasil, a prática de notificação de acidentes de trabalho ainda carece de incentivo e de maior fiscalização, pois a literatura mostra casos de não notificação dos acidentes ${ }^{(6)}$. Diante desta constatação, foi criado pelo governo o SINAN para o registro de acidentes de trabalho com exposição a material biológico na tentativa de conhecer a real situação destes acidentes e obter dados para subsidiar futuras ações preventivas.

Os modelos preventivos em vigor não são respeitados e as medidas adotadas pelas empresas estão direcionadas para o risco efetivo e não para a prevenção dos acidentes. As medidas necessárias para tornar um ambiente de trabaIho mais adequado ao bom desempenho do trabalhador e que, por sua vez, assegure sua capacidade física e mental é negligenciado(1-8).

Diante do contexto apresentado, foram elaboradas as seguintes questões de pesquisa: como pode ser compreendido o conceito de risco desenvolvido pelos trabalhadores do hospital? Quais as interpretações simbólicas desenvolvidas por estas pessoas que vivem cotidianamente em contato com objetos perfurocortantes que materializam este risco a acidentes?

\section{OBJETIVOS}

$\checkmark \quad$ Levantar os acidentes de trabalho entre profissionais de saúde e estudantes atuantes no Hospital Universitário de Brasília;

$\checkmark$ Identificar os riscos ocupacionais das unidades do CTI adulto e Lavanderia do hospital;

$\checkmark$ Compreender como os trabalhadores percebem e interpretam os riscos do ambiente e dos instrumentos de trabaIho e os acidentes com material perfurocortante.

\section{MÉTODOS}

Trata-se de pesquisa exploratória, descritiva, com análise qualitativa e quantitativa de dados, realizada no Hospital Universitário de Brasília após aprovação do CEP, sob o registro de número 130/09 de 11/02/2009. Todos os sujeitos assinaram o Termo de Consentimento Livre e Esclarecido.

$\mathrm{O}$ número de acidentes de trabalho com material perfurocortante foi tratado com uso do teste estatístico Qui-quadrado $\left(\chi^{2}\right)$ de independência, realizado no SPSS 15.0, para verificar associação com a variável local de trabalho (CTI adulto e Lavanderia); os dados relativos à compreensão do risco pelo trabalhador no ambiente hospitalar foram tratados por meio de abordagem qualitativa apoiada no referencial das Ciências Humanas ${ }^{(8)}$.

Os dados foram coletados em três etapas, sendo a primeira correspondente ao levantamento dos acidentes de trabalho ocorridos no HUB realizada no período de 03 a 14 de maio de 2010.

$1^{\text {a }}$ etapa: Observação de campo com a finalidade de realizar um diagnóstico dos riscos (físicos, químicos, biológicos, ergonômicos e de acidentes), visão do fluxo do trabalho, funcionamento do maquinário, número de trabalhadores, exposição dos trabalhadores aos riscos, problemas de saúde mais comuns, uso de equipamentos de proteção individual e presença de equipamentos de proteção coletiva. Para os riscos quantificáveis, foram utilizados aparelhos de medição como: decibelímetro (ruído), luxímetro (iluminância) e termoanemômetro (temperatura, umidade e velocidade do ar), em 30/03/10.

$\mathbf{2}^{\mathbf{a}}$ etapa: Aplicação dos questionários semiestruturados com perguntas abertas e fechadas, realizada entre os dias 20/04//2010 e 20/05/2010, para 105 trabalhadores, sendo 56 da lavanderia e 49 do $\mathrm{CTI}$ adulto, correspondentes, respectivamente, a $98,2 \%$ e $83,1 \%$ do total de trabalhadores lotados nas referidas unidades, com os seguintes critérios de inclusão: os trabalhadores que aceitaram participar do estudo e em efetivo exercício nas unidades.

$3^{\text {a }}$ etapa: Levantamento de dados realizados no setor de Medicina do Trabalho por meio de instrumento utilizado para notificação de acidentes conhecido como: Comunicação Interna de Acidentes de Trabalho (CIAT), para verificar situação de saúde, caracterizar os acidentes e doenças dos trabalhadores.

\section{RESULTADOS}

Riscos ocupacionais físicos, químicos, biológicos, ergonômicos e riscos de acidentes foram identificados no CTI e na lavanderia por meio da técnica de mapa de risco. No CTI todos os valores encontrados estiveram dentro da normalidade, já na lavanderia, os níveis de ruído ultrapassaram $85 \mathrm{~dB}(\mathrm{~A})$ para uma jornada de trabalho de 12 horas (próximo às calandras e máquinas de lavar).

$\mathrm{Na}$ avaliação de temperatura ambiente, os valores apresentados ficaram de $30.0^{\circ} \mathrm{C}$ a $34.0^{\circ} \mathrm{C}$, em desacordo com a NR $n^{\circ} 17$, sendo importante buscar medidas que se tornem mais propício para o trabalho (climatização, exaustores, abertura de ventilação natural, a depender do setor). Quanto a velocidade do ar, atingiu no setor de calandras $1,3 \mathrm{~m} / \mathrm{s}$ estando em desacordo com a norma e a umidade do ar, ficou em torno de $45 \%$ (acima do normal). 
Quanto aos acidentes de trabalho entre profissionais de saúde e estudantes atuantes no Hospital Universitário de Brasília, a Tabela 1 reúne os resultados obtidos.

Tabela 1 - Distribuição de acidentes de trabalho sofridos pelos funcionários segundo local de trabalho no HUB. Brasília, 2010.

\begin{tabular}{c|cc|c|c}
\hline \multirow{2}{*}{ Acidentes de trabalho } & \multicolumn{4}{|c}{ Local de trabalho } \\
\cline { 2 - 5 } & \multicolumn{2}{|c|}{ Lavanderia } & \multicolumn{3}{c}{ CTI } \\
\cline { 2 - 5 } & $\mathbf{f}$ & $\%$ & $\mathbf{f}$ & $\%$ \\
\hline Sim & 11 & 19,6 & 13 & 26,5 \\
Não & 45 & 80,4 & 36 & 73,5 \\
\hline Total & 56 & 100,0 & 49 & 100,0 \\
\hline
\end{tabular}

Foi realizado o teste de Qui-quadrado $\left(\chi^{2}\right)$ para saber se havia relação entre as variáveis acidentes de trabalho e local de trabalho (Lavanderia e CTI). O p-valor encontrado foi igual a 0,33. Logo, como o P-valor $>0,05$, ao nível de significância de $5 \%$, não se rejeita a hipótese de independência entre essas as variáveis, ou seja, os acidentes não estão relacionados com o local de trabalho.

Dos questionários aplicados na lavanderia, 58,9\% dos trabalhadores apresentam problemas de saúde, 19,6\% já sofreram acidentes de trabalho, 17,9\% notificaram, 98,2\% tiveram treinamento sobre prevenção de acidentes e 83,9\% conhecem o mapa de riscos. No CTI, 36,7\% apresentam problemas de saúde, 26,5\% já sofreram acidentes de trabalho, 24,5\% notificaram, 34,7\% tiveram treinamento sobre prevenção de acidentes e 12,2\% conhecem o mapa de riscos.

Foram analisados todos os prontuários dos sujeitos envolvidos no intuito de levantar possíveis causas de doenças ocupacionais, não sendo encontrado nenhum caso que evidenciasse doença adquirida em decorrência das atividades laborativas.

Por outro lado, os acidentes de trabalho analisados por meio das CIATs com materiais biológicos/perfurocortantes entre os anos de 2003 e 2009, mostraram nove acidentes no CTI adulto e apenas três na lavanderia. Acreditamos que tenha havido subnotificação ou não notificação de alguns acidentes em ambos os setores. Concluímos que os trabalhadores da lavanderia são mais conscientizados quanto às precauções e à adoção de medidas preventivas quanto ao uso de EPIs, somado aos treinamentos que tiveram.

Um dos pontos principais também abordados durante a pesquisa foram os problemas de saúde apontados pelas profissionais que atuam nos ambientes pesquisados; na tabela que se segue temos os dados referentes à percepção dos trabalhadores com relação ao risco no local de trabalho.

Tabela 2 - Perfil dos trabalhadores da lavanderia e CTI adulto do HUB segundo problemas de saúde. Brasília, 2010.

\begin{tabular}{c|cc|cc}
\hline \multirow{2}{*}{ Problemas de saúde } & \multicolumn{4}{|c}{ Local de trabalho } \\
\cline { 2 - 5 } & \multicolumn{2}{|c}{ Lavanderia } & \multicolumn{3}{c}{ CTI } \\
\cline { 2 - 5 } & $\mathbf{f}$ & $\%$ & $\mathbf{f}$ & $\%$ \\
\hline Sim & 33 & 58,9 & 18 & 36,7 \\
Não & 23 & 41,1 & 31 & 63,3 \\
\hline Total & 56 & 100,0 & 49 & 100,0 \\
\hline
\end{tabular}

Nessa tabela, os problemas de saúde não são vinculados ao local de trabalho sendo que $36,7 \%$ disseram ter problemas vinculados com a atividade laboral. Este resultado pode ser pensado como uma resposta a familiarização com as atividades, naturalização das atividades desenvolvidas e a negação de que eles poderiam causar as doenças.

Em estudo anterior, foi constatado que os acidentes de trabalho com exposição ocupacional a material biológico são os de maior ocorrência entre os trabalhadores deste mesmo hospital ${ }^{(11)}$. Estes tipos de acidentes também tem grandes ocorrências em outros hospitais ${ }^{(12)}$.

Na Tabela 3 são apresentados dados sobre as doenças adquiridas após a admissão no hospital.

Tabela 3 - Perfil dos trabalhadores da lavanderia e CTI adulto do HUB segundo doenças adquiridas após admissão. Brasília, 2010.

\begin{tabular}{c|cc|cc}
\hline \multirow{2}{*}{$\begin{array}{c}\text { Doenças adquiri- } \\
\text { das após admissão }\end{array}$} & \multicolumn{4}{|c}{ Local de trabalho } \\
\cline { 2 - 5 } & \multicolumn{2}{|c}{ Lavanderia } & \multicolumn{3}{c}{ CTI } \\
\cline { 2 - 5 } & $\mathbf{f}$ & $\%$ & $\mathbf{f}$ & $\%$ \\
\hline Sim & 05 & 8,9 & 08 & 16,3 \\
Não & 51 & 91,1 & 41 & 83,7 \\
\hline Total & 56 & 100,0 & 49 & 100,0 \\
\hline
\end{tabular}

Embora fique claro pelos dados quantitativos que as pessoas não associam suas doenças com o local de trabalho, é importante entender que muitas vezes elas não têm uma compreensão de como o trabalho afeta a saúde. Aos olhos de uma pessoa leiga, uma doença como cefaléia crônica, as doenças plurimetabólicas e psicossomáticas (ansiedade, depressão) nem sempre seriam vinculadas com um processo contínuo de estresse no ambiente de trabalho.

As tabelas apresentadas a seguir se referem às notificações de risco e dos acidentes de trabalho.

Tabela 4 - Perfil dos trabalhadores da lavanderia e CTI adulto do HUB segundo conscientização da notificação de acidentes de trabalho. Brasília, 2010.

\begin{tabular}{c|cc|cc}
\hline \multirow{2}{*}{$\begin{array}{c}\text { Conscientização } \\
\text { da notificação }\end{array}$} & \multicolumn{4}{|c}{ Local de trabalho } \\
\cline { 2 - 5 } & \multicolumn{2}{|c|}{ Lavanderia } & \multicolumn{3}{c}{ CTI } \\
\cline { 2 - 5 } & $\mathbf{f}$ & $\%$ & $\mathbf{f}$ & $\%$ \\
\hline Sim & 47 & 83,9 & 43 & 87,8 \\
Não & 09 & 16,1 & 06 & 12,2 \\
\hline Total & 56 & 100,0 & 49 & 100,0 \\
\hline
\end{tabular}

Tabela 5 - Perfil dos trabalhadores da lavanderia e CTI adulto do HUB segundo realização de notificação de acidentes de trabalho. Brasília, 2010.

\begin{tabular}{c|cc|cc}
\hline \multirow{2}{*}{$\begin{array}{c}\text { Notificação de } \\
\text { acidentes }\end{array}$} & \multicolumn{4}{|c}{ Local de trabalho } \\
\cline { 2 - 5 } & \multicolumn{2}{|c}{ Lavanderia } & \multicolumn{3}{c}{ CTI } \\
\cline { 2 - 5 } & $\mathbf{f}$ & $\%$ & $\mathbf{f}$ & $\%$ \\
\hline Sim & 10 & 17,9 & 12 & 24,5 \\
\hline Não & 46 & 82,1 & 37 & 75,5 \\
\hline Total & 56 & 100,0 & 49 & 100,0 \\
\hline
\end{tabular}


Observa-se que a ausência de notificação dos que já sofreram acidentes é alta. No entanto, faz-se necessário aprofundar a pesquisa para compreender o que fez com que essas pessoas negligenciassem o risco e que tipo de risco foi negligenciado, considerando que em uma situação de risco com material contaminado por vírus ou bactéria com alto poder de contaminação e proliferação poderia contaminar todo o hospital e até mesmo levar a um quadro que afete a saúde pública(10-13).

Quanto à capacitação dos trabalhadores, a Tabela 6 mostra os dados relativos aos treinamentos recebidos.

Tabela 6 - Perfil dos trabalhadores da lavanderia e CTI adulto do HUB segundo treinamento sobre prevenção de acidentes de trabalho. Brasília, 2010.

\begin{tabular}{c|c|c|c|c}
\hline \multirow{2}{*}{$\begin{array}{c}\text { Treinamento sobre } \\
\text { prevenção de } \\
\text { acidentes }\end{array}$} & \multicolumn{4}{|c}{ Local de trabalho } \\
\cline { 2 - 5 } & \multicolumn{2}{|c}{ Lavanderia } & \multicolumn{2}{c}{ CTI } \\
\cline { 2 - 5 } & $\mathbf{f}$ & $\%$ & $\mathbf{f}$ & $\%$ \\
\hline Sim & 55 & 98,2 & 17 & 34,7 \\
Não & 01 & 1,8 & 32 & 65,3 \\
\hline Total & 56 & 100,0 & 49 & 100,0 \\
\hline
\end{tabular}

Salienta-se a importância da participação dos profissionais no processo de treinamento e o desenvolvimento de um sentimento de responsabilidade com a segurança, mas não como um mecanismo de culpabilização e sim como um processo de reconhecimento de pertencimento de um determinado ambiente de trabalho em que todos são responsáveis pela segurança.

Sobrecarga de trabalho, fatalidade, culpa própria, negligência e precariedade das condições de trabalho foram citadas como as causas mais frequentes de lesões que ocasionaram acidentes de trabalho no período de julho de 2002 a julho de 2003. Houve predominância do gênero feminino, por representar a maior força de trabalho nos hospitais, na categoria de auxiliares de enfermagem, no período da manhã, quando é realizada a maior parte dos procedimentos (medicações, cirurgias, coleta de material para exames, tendo os quirodáctilos as partes dos corpos mais atingidas ${ }^{(14)}$.

Dados da tabela 6 mostram que praticamente todos fizeram o processo de treinamento. Embora estes dados sejam relevantes, eles entram em contradição com o índice de acidentes não notificados.

Essa contradição está diretamente vinculada com o âmbito subjetivo do indivíduo, que determina como cada pessoa vai aplicar no seu dia-a-dia os conhecimentos adquiridos nos cursos de capacitação. Se o processo de compreensão do risco engloba os âmbitos subjetivo e coletivo ao mesmo tempo, são necessárias estratégias cotidianas que envolvam todos para que esses dois processos tenham significado e possam ser realizados juntos para minimizar os riscos.

Em pesquisa realizada num hospital público do Distrito Federal, os autores identificaram como fatores desencadeantes de acidentes a dupla jornada de trabalho, a falta de treinamento e capacitação, os ambientes físicos inadequados, a falta de material adequado tanto em qualidade como em quantidade, a ausência de manutenção preventiva de equipamentos, o grande número de procedimentos invasivos e o quantitativo insuficiente de trabalhadores, gerando sobrecarga de trabalho ${ }^{(15)}$

Os dados comprovam como o risco de acidentes nos ambientes laborais dos hospitais é elevado. Considerando todos os dados apresentados, podemos destacar a importância da criação de instrumentos educacionais que contribuam para a capacitação cotidiana dos profissionais (incluindo os estagiários).

Considerando que o risco faz parte do ambiente de trabaIho do hospital, devem ser criados mecanismos que façam com que instrumentos como o "Mapa de Riscos" e os equipamentos de proteção individual e coletivo façam parte do cotidiano de todos que atuam nesse ambiente de trabalho. Portanto, a criação de processos contínuos de aprendizagem coletivos, podem ser mecanismos fundamentais para consolidação da conscientização destes profissionais da saúde.

No nosso entendimento, alguns trabalhadores dos setores do estudo enfrentam os riscos com maior seriedade, adotando as medidas universais de biossegurança, enquanto que, outros negligenciam os usos de EPIS, até mesmo técnicas simples como lavagem das mãos, reencape de agulhas, descarte de objetos perfurocortantes em locais inapropriados, deixando a saúde e a segurança no trabalho relegadas a segundo plano e se preocupando apenas nos direitos de receberam o adicional de insalubridade.

Os resultados demonstraram que apenas a avaliação da iluminação estava dentro de todos os padrões exigidos pelas normas técnicas, embora a variação de pequenos desajustes nos outros quesitos não apontem problemas separadamente, numa perspectiva conjuntural, esses valores mostram que o espaço traz elementos que tornam o ambiente insalubre e que podem afetar de forma direta o desempenho do trabalho.

\section{CONSIDERAÇÕES FINAIS}

O ambiente de trabalho dos hospitais é um espaço que esconde riscos iminentes tanto para as pessoas quanto para o meio ambiente e, neste sentido, deve ser objeto de constante processo de avaliação e controle.

Mesmo que façamos um recorte específico com a análise dos acidentes com perfurocortantes, ainda assim, fica clara a necessidade de um controle do ambiente do trabalho e dos trabalhadores que cotidianamente atuam nesse espaço.

Dois mecanismos de controle de risco foram apontados com recursos essenciais para a efetiva diminuição dos riscos: o mapa de riscos ambientais e os equipamentos de proteção (individual e coletivos). Para efetiva consolidação dessas estratégias, o HUB deve desenvolver vários instrumentos educativos que possibilitem consolidação das práticas de segurança.

Um dos mecanismos mais utilizados pelas instituições públicas na área de capacitação continuada é a educação à distância. Essa modalidade de educação possibilitaria que os profissionais que atuam no Hospital pudessem reciclar seus conhecimentos sobre segurança no trabalho e criar laços de companheirismo que possibilitariam, por sua vez, o desenvolvimento de novas redes de solidariedade dentro do ambiente laboral.

É importante salientar que o ambiente de trabalho é um espaço onde são vivenciadas experiências subjetivas e coletivas. Nesse sentido, quanto maior o sentimento de pertencimento a um coletivo, maior será a preocupação em mantê-lo protegido dos riscos. 


\section{REFERÊNCIAS}

1. Brasil. Lei $n^{\circ}$. 8.213 de 24 de julho de 1991. Dispõe sobre os Planos de Benefícios da Previdência Social e dá outras providências. Diário Oficial da União 27 jul 1991.

2. Porto MFS. Análise de riscos nos locais de trabalho: conhecer para transformar. Cad Saúde Trab [periódico na internet]. 2000 [acesso em 10 fev 2010] Disponível em: <http:// normasregulamentadoras.files.wordpress.com/2008/06/ riscos_trabalho.pdf >

3. Ministério do Trabalho e Emprego (Brasil). Portaria $\mathrm{n}^{\circ}$. 3.214 de 8 de junho de 1978. Aprova as Normas Regulamentadoras - NR - do Capítulo V, Título II, da consolidação das leis do trabalho, relativas a Segurança e Medicina do Trabalh [portaria na internet]. Diário Oficial da União 6 jul 1978. [acesso em 10 fev 2010] Disponível em: <http:// www1.pucminas.br/imagedb/documento/DOC_DSC_ NOME_ARQUI20081104143646.pdf>

4. Mattos UA, Freitas NBB. Mapa de Riscos no Brasil: As limitações da aplicabilidade de um modelo operário. Cad Saúde Pública 1994;10(2):251-8.

5. Almeida G, Guena RM. A saúde do trabalhador em jogo [artigo na internet] Salvador. [acesso em 10 fev 2011] Disponível em: < http://www.pstu.org.br/nacional_materia. asp? $\mathrm{id}=11111$ \&ida $=0>$

6. Napoleão AA, Robazzi MLCC, Marziale MHP, Hayashida M. Causas de subnotificação de acidentes do trabalho entre trabalhadores de enfermagem. Rev Latino-Am Enferm 2000;8(3):119-20.

7. Costa NL. Capoeira, trabalho e educação. Bahia. Dissertação [Mestrado em Educação]- Universidade Federal da
Bahia. Faculdade de Educação; 2007.

8. Douglas M. La aceptabilidad del riesgo según las Ciencias Sociales. Barcelona: Paidós; 1996.

9. Lurján JLS. Reseña de "La aceptabilidad del Riesgo según las Ciencias Sociales" de Mary Douglas. Relaciones 2004;25(997).

10. Marziale MHP, organizador. Repat-USP. Rede Eletrônica de Prevenção de Acidentes de Trabalho com exposição a material biológico [monografia na internet] 2003. [acesso em 10 fev 2011] Disponível en: < http://repat.eerp.usp. $\mathrm{br} />$

11. Marziale MHP, Silva EJ, Hass VJ, Robazzi MLC. Acidentes com material biológico em hospital de Rede de Prevenção de Acidentes de Trabalho - REPAT. Rev Bras Saúde Ocup 2007;32(115):109-19.

12. Marziale MHP, Rodrigues CM. A produção científica sobre os acidentes de trabalho com material perfurocortante entre trabalhadores de enfermagem. Rev Latino-Am Enferm 2002;10(4):571-7.

13. Brevidelli MM, Cianciarulo TI, Análise dos acidentes com agulhas em um hospital universitário: situações de ocorrência e tendências. Rev Latino-Am Enfermagem 2002;10(6):780-6.

14. Pinho DLM, Rodrigues CM, Gomes GP. Perfil dos acidentes de trabalho no Hospital Universitário de Brasília. Rev Bras Enferm 2007;60(3):291-4.

15. Ribeiro EJG, Shimizu HE. Acidentes de trabalho com trabalhadores de enfermagem. Rev Bras Enferm 2007;60(5):535-40. 